\title{
Laser-plasma and stellar astrophysics spectroscopy
}

\author{
Christian G. Parigger \\ Physics and Astronomy Department, University of Tennessee, \\ University of Tennessee Space Institute, Center for Laser Applications, \\ 411 B.H. Goethert Pkwy, Tullahoma, TN 37388, USA \\ (E-mail: cparigge@tennessee.edu)
}

Received: June 6, 2019; Accepted: July 22, 2019

\begin{abstract}
This work examines time-resolved spectroscopy of the Balmer series lines in laboratory settings and applies results for interpretation of spectra from astrophysical objects. White dwarf stars reveal a variety of atomic or molecular line shapes in absorption depending on the age of these cooling stars. Laboratory investigations show emission profiles that are correlated with astrophysical records. For the hydrogen beta line, $\mathrm{H} \beta$, and the hydrogen delta line, $\mathrm{H} \delta$, of the Balmer series, line width and peak separation are indicators of electron density. In addition, widths of the hydrogen alpha line, $\mathrm{H} \alpha$, and hydrogen gamma line, $\mathrm{H} \gamma$, can be utilized along with $\mathrm{H} \alpha$ shifts and $\mathrm{H} \beta$ dipshifts. Of interest are experiments and analysis of laser- and astrophysical white-dwarfplasma spectral line shapes, especially comparisons of transient laser-plasmawith gravitational white-dwarf- redshifts. Current and future research aspects include modeling of white-dwarf atmospheres for explanation of the very details of the astrophysical line shapes.

Key words: atomic and molecular spectroscopy - time-resolved spectroscopy - laser plasma - laser-induced optical breakdown - stellar astrophysics spectra

- white dwarf stars - hydrogen
\end{abstract}

\section{Introduction}

Recorded emission spectra of astrophysical objects usually reveal absorption lines and bands. Analysis of the spectra allows one to make inferences about the star under investigation. In the visible region of the electromagnetic spectrum, $\mathrm{H} \beta$ is of primary interest in the characterization of white dwarf (WD) stars. The temperature of the closest WD to earth, Sirius B ( $\alpha$ CMa B), is of the order of $30 \mathrm{kK}$. Sirius B accompanies the brightest star Sirius A as seen from earth. However, instead of hydrogen absorption spectra, molecular carbon Swan absorption spectra are recorded for cooler WD's at a temperature of the order of $8 \mathrm{kK}$, viz. Procyon B ( $\alpha$ CMi B), companion of the fifth brightest star seen from earth, namely, Procyon A. In the laboratory, hydrogen Balmer series emission spectra are recorded, including determination of temperature using the line-tocontinuum ratio. The mass of WD stars is typically of the order of the mass of 
the sun $\left(\mathrm{M}_{\odot}\right)$, but the WD radius is about the size of the earth, or 1/100 times that of the sun $\left(0.01 \mathrm{R}_{\odot}\right)$.

This work communicates experiments of laser plasma in hydrogen, gaseous mixtures of hydrogen and nitrogen, and air at standard ambient temperature and pressure. Ultra-pure hydrogen and gaseous mixtures experiments employ a laboratory cell for measurement of the Balmer series lines. Diatomic molecular recombination spectra are also recorded in laser-plasma of gaseous mixtures and laser-ablation plasma.

In recent hydrogen experiments, measurements address the first four Balmer series hydrogen lines - early in the plasma decay, neutral and ionized nitrogen lines are identified and appear first as the plasma cools. Plasma characteristics for time delays up to $0.275 \mu$ s were previously discussed albeit for exclusively hydrogen gas at $0.75 \mathrm{~atm}$ and ambient temperature (Parigger et al., 2018). In this work, optical breakdown plasma is generated in a 1:1 hydrogen:nitrogen mixture at ambient temperature and 0.27-atm pressure (Parigger, 2019). Plasma diagnosis is applied for time delays in the range of $0.25 \mu \mathrm{s}$ to $3.25 \mu \mathrm{s}$. Nitrogen contributes to the breakdown plasma seven times more electrons than hydrogen in cases of full ionization. Time-resolved spectroscopy records emitted radiation with spatial radiation along the slit height for the $\mathrm{H} \alpha, \mathrm{H} \beta, \mathrm{H} \gamma$, and $\mathrm{H} \delta$ lines.

Laser-plasma diagnosis and characterization enjoy recent and significant interests in part due to needs for determination of chemical composition of materials. Specifically, laser-induced breakdown spectroscopy (LIBS, Miziolek et al., 2018; Singh \& Thakur, 2019) is preferred over spark-induced breakdown spectroscopy for chemical analysis. In stellar astrophysics however, hydrogen spectroscopy is essential for determination of atmospheres of stars, and related, for determination of the atmospheric composition of planets or exo-planets with established molecular data-bases (Tennyson \& Yurchenko, 2012).

Typically, the micro-plasma is initiated with of the order of 10-nanosecond laser radiation and is recorded with time-resolved plasma spectroscopy (Kunze, 2009 ) for time delays up to a few $\mu$ s for the recording of atomic hydrogen spectra, and for time delays of a few 10's of $\mu$ s for molecular spectra. The accuracy of the $\mathrm{H} \beta$ line is better than that of the $\mathrm{H} \alpha$ line (Wiese, 1965) and recommendations for plasma spectroscopy include addition of trace amounts of hydrogen to accomplish accurate diagnosis (Wiese, 1965). Challenges in comparisons of laboratory and astrophysical plasma-spectra have been reviewed recently (Halenka et al., 2015), i.e., comparisons of $\mathrm{H} \beta$ line shapes of micro-plasma recorded with time-resolved emission spectroscopy and of $\mathrm{H} \beta$ line shapes in astrophysical white dwarf macro-plasma absorption spectra that are measured continuously at various observatories. 


\section{Experimental details}

Laboratory laser-plasma measurements employ a pulsed, Q-switched, Nd:YAG laser device (Q-Smart 850 Quantel laser, USA) operated at a pulse-width of $6 \mathrm{~ns}$ and a pulse energy of $850 \mathrm{~mJ}$ at the wavelength of $1064 \mathrm{~nm}$. Laser-induced optical breakdown is generated by focusing $150 \mathrm{~mJ}$ per pulse of ir fundamental radiation to achieve of the order of $1 \mathrm{TW} / \mathrm{cm}^{2}$ in a cell containing a $1: 1$ mixture of hydrogen and nitrogen, introduced at a pressure of 0.135 atm each after establishing a nominal mercury-diffusion-pump vacuum in the cell of the order $10^{-5}$ mbar. A crossed CzernyTurner spectrometer (Jobin Yvon $0.64 \mathrm{~m}$ triple spectrometer, France) of 0.64-nm focal length disperses the emission spectra. The pulsed radiation is focused into the cell with the beam propagating from the top and parallel to the vertical $100 \mu \mathrm{m}$ spectrometer slit (Parigger, 2019). Further details of the experimental arrangement that is similar to the ultrapure hydrogen experiments were communicated previously (Parigger et al., 2014, 2018, 2019a,b).

The spectral resolution amounts to $0.1 \mathrm{~nm}$ for the selected $1200 \mathrm{~g} \mathrm{~mm}^{-1}$ holographic grating following corrections of the wavelength variation along the slit direction. Of the order of 24-nm spectral coverage for the 1024 pixels along the wavelength-dimension, the 0.1-nm resolution corresponds to an instrumentprompt width of on-average 4.25 pixels. Conversely, grouping four pixels along the slit dimensions corresponds to a spatial resolution of $54.4 \mu \mathrm{m}$ as the pixel area amounts to $13.6 \mu \mathrm{m} \times 13.6 \mu \mathrm{m}$.

For 13 selected time delays from $0.25 \mu \mathrm{s}$ to $3.25 \mu \mathrm{s}$ and $0.025 \mu$ s gate-widths, micro-plasma data are captured. Of interest are the peak-separation and width of $\mathrm{H} \delta$ and width of $\mathrm{H} \gamma$ for electron densities in the range of 0.1 to $1 \times 10^{17} \mathrm{~cm}^{-3}$, and comparisons with $\mathrm{H} \beta$ and $\mathrm{H} \alpha$ diagnostics. Integral inversion interrogates the lateral spatial distribution of the recorded line-of-sight plasma expansion.

\section{Results}

\subsection{Line-of-sight measurements}

A total of 52 two-dimensional data sets are recorded for the four $\mathrm{H} \alpha, \mathrm{H} \beta, \mathrm{H} \gamma$, and $\mathrm{H} \delta$ Balmer series lines, including several nitrogen and ionized nitrogen lines that occur especially for time delays in the range of $0.25-0.75 \mu$ s following plasma initiation. Fig. 1 portrays spectral radiance, using grey-scale representation, of individually scaled maps of the four hydrogen lines for a time delay of $3 \mu \mathrm{s}$.

Features of Fig. 1 include expected Stark-width behavior (Holtsmark, 1919; Greene, 1961; Griem, 1974; Oks, 2017): (i) full-widths at half maximum increase from the $\mathrm{H} \alpha$ line to the $\mathrm{H} \delta$ line; (ii) the maximum signals decrease from $\mathrm{H} \alpha$ to $\mathrm{H} \delta$; (iii) peak-separation of the $\mathrm{H} \beta$ (Ivković et al., 2015) and $\mathrm{H} \delta$ lines, and $\mathrm{H} \beta$ dipshifts (Halenka et al., 1989; Mijatović et al., 1991). The occurrence of dips for 
(a)

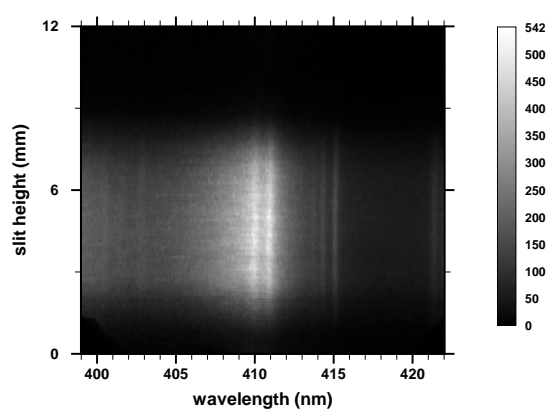

(c)

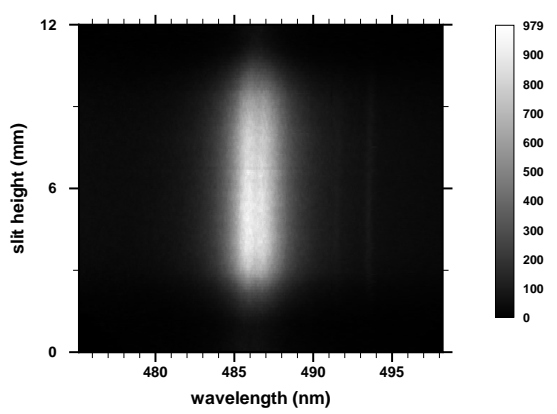

(b)

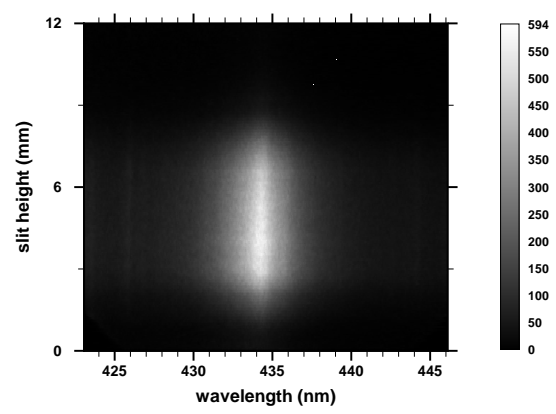

(d)

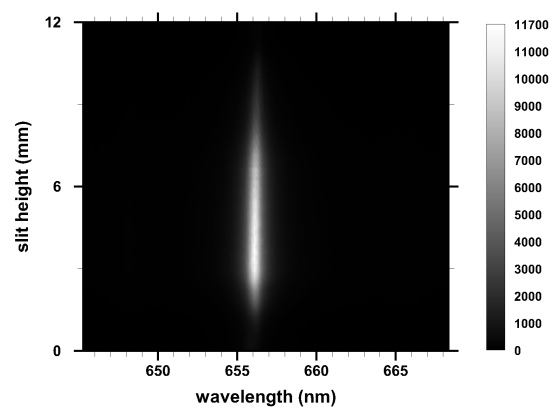

Figure 1. Balmer series hydrogen lines versus wavelength at $3-\mu$ s time delay, $0.025-\mu$ s gate. (a) $\mathrm{H} \delta$, (b) $\mathrm{H} \gamma$, (c) $\mathrm{H} \beta$, and (d) $\mathrm{H} \alpha$.

$\mathrm{H} \beta$ and $\mathrm{H} \delta$ are not due to self-absorption for electron densities of the order of $10^{17} \mathrm{~cm}^{-3}$ - predictions of Stark effects on $\mathrm{H} \beta$ and $\mathrm{H} \delta$ with associated absence of line-center components are part of the reason for E. Schrödinger (Schrödinger, 1926) receiving the Nobel Prize, shared with P. Dirac (Physics Nobel Prize, 1933).

In the images, detector dark-counts or background contributions are subtracted, sensitivity calibrations by reference to standard lamps are applied, and linear wavelength calibration is performed with penray lamps and by using calibrated spectrometer dials. The image of the spectrometer slit usually is slightly curved near the edges, consequently, wavelength calibrations are performed for each spectrum recorded along the slit. For the experiments reported here, four vertical pixels are combined to increase sensitivity requiring 256 individual wave- 
length calibrations for each of four spectrometer positions. The 256 recorded, wavelength calibrated Balmer series spectra are slightly wavelength-shifted and interpolated for display of the data versus slit height and wavelength position. In other words, the displayed maps are corrected for the spectrometer-related wavelength variations along the slit height. Consequently, the maps in Fig. 1 are primed for analysis that employs integral inversion of the recorded line-of-sight data. However, data are first analyzed to determine variations and averages along the slit.

Analysis of the laboratory emission spectra utilizes established empirical formulae for $\mathrm{H} \alpha$ and $\mathrm{H} \beta$ (Parigger et al., 2018). For $\mathrm{H} \alpha$, the width, $\Delta \lambda_{\alpha}$, and shift, $\delta \lambda_{\alpha}$, are indicators for electron density,

$$
\begin{gathered}
\Delta \lambda_{\alpha}[\mathrm{nm}]=1.3\left(\frac{\mathrm{N}_{\mathrm{e}}\left[\mathrm{cm}^{-3}\right]}{10^{17}}\right)^{0.64 \pm 0.03}, \\
\delta \lambda_{\alpha}[\mathrm{nm}]=0.055\left(\frac{\mathrm{N}_{\mathrm{e}}\left[\mathrm{cm}^{-3}\right]}{10^{17}}\right)^{0.97 \pm 0.03} .
\end{gathered}
$$

Analysis of $\mathrm{H} \beta$ offers three indicators of electron density: Width, $\Delta \lambda_{\beta}$, peak separation, $\delta \lambda_{\beta-\mathrm{ps}}$, and dip shift, $\delta \lambda_{\beta-\mathrm{ds}}$,

$$
\begin{aligned}
\Delta \lambda_{\beta}[\mathrm{nm}] & =4.5\left(\frac{\mathrm{N}_{\mathrm{e}}\left[\mathrm{cm}^{-3}\right]}{10^{17}}\right)^{0.71 \pm 0.03}, \\
\delta \lambda_{\beta-\mathrm{ps}}[\mathrm{nm}] & =1.3\left(\frac{\mathrm{N}_{\mathrm{e}}\left[\mathrm{cm}^{-3}\right]}{10^{17}}\right)^{0.61 \pm 0.03} \\
\delta \lambda_{\beta-\mathrm{ds}}[\mathrm{nm}] & =0.14\left(\frac{\mathrm{N}_{\mathrm{e}}\left[\mathrm{cm}^{-3}\right]}{10^{17}}\right)^{0.67 \pm 0.03}
\end{aligned}
$$

The $\mathrm{H} \beta$ dipshift allows one to measure electron density (Parigger et al., 2019a) up to the $\mathrm{H} \beta$ Inglis-Teller limit (Inglis \& Teller, 1939) of $60 \times 10^{17} \mathrm{~cm}^{-3}$. However, $\mathrm{H} \beta$ is preferred in a variety of astrophysics data-reduction efforts for electron densities of the order of $10^{17} \mathrm{~cm}^{-3}$.

\section{2. $\mathbf{H} \delta$ and $\mathbf{H} \gamma$ line profiles}

The analysis of the $\mathrm{H} \delta$ and $\mathrm{H} \gamma$ data rely on computer simulations (Gigosos et al., 2003), and in this work, on published Stark tables (Griem, 1974) that only show electron-density data in the range of 0.1 to $1 \times 10^{17} \mathrm{~cm}^{-3}$ for a temperature of $20 \mathrm{kK}$. From the Stark tables, $\mathrm{H} \delta$ and $\mathrm{H} \gamma$ line-profiles can be constructed for electron densities, $\mathrm{N}_{\mathrm{e}}$, of 0.1 and $1 \times 10^{17} \mathrm{~cm}^{-3}$. Subsequently, $\mathrm{H} \delta$ and $\mathrm{H} \gamma$ full-width half maximum (FWHM) can be determined for plasma diagnosis, moreover, $\mathrm{H} \delta$ invites the use of peak separation (PS) as a diagnostic tool. 
From log-log fitting of $\mathrm{H} \gamma$ and $\mathrm{H} \delta$ data in the range of 0.1 to $1 \times 10^{17} \mathrm{~cm}^{-3}$, one obtains for $\mathrm{H} \delta \mathrm{FWHM}, \Delta \lambda_{\delta}$,

$$
\Delta \lambda_{\delta}[\mathrm{nm}]=10\left(\frac{\mathrm{N}_{\mathrm{e}}\left[\mathrm{cm}^{-3}\right]}{10^{17}}\right)^{0.67}
$$

and the $\mathrm{H} \delta$ peak-separation, $\Delta \lambda_{\delta-\mathrm{ps}}$, in the range of 0.1 to $1 \times 10^{17} \mathrm{~cm}^{-3}$ amounts to

$$
\Delta \lambda_{\delta-\mathrm{ps}}[\mathrm{nm}]=2.0\left(\frac{\mathrm{N}_{\mathrm{e}}\left[\mathrm{cm}^{-3}\right]}{10^{17}}\right)^{0.62} .
$$

The $\mathrm{H} \delta$ line shows peak separations just like the $\mathrm{H} \beta$ line. From log-log fitting, one obtains for $\mathrm{H} \gamma \mathrm{FWHM}, \Delta \lambda_{\gamma}$,

$$
\Delta \lambda_{\gamma}[\mathrm{nm}]=6.0\left(\frac{\mathrm{N}_{\mathrm{e}}\left[\mathrm{cm}^{-3}\right]}{10^{17}}\right)^{0.72} .
$$

Analysis of laser-plasma details requires both spatial and temporal resolutions. However, the average of the spectra along the slit-dimension are investigated first.

Fig. 2 displays signatures of the first four members of the Balmer series. The spectra are averaged over 220 individual spectral lines, and are also averaged over 100 accumulated laser-plasma events. Considering an average over 22,000 spectra, and with the nominal square-root reduction in variance by a factor of 148 , further filtering of the data is not necessary.

\subsection{Abel-inverted spectral maps}

The recorded line-of-sight data can be further processed using inverse integral transforms in order to examine the spatial distribution of the expanding plasma. Motivation derives from recorded shadow-graphs depicted in Fig. 3.

Abel-inverted, first four Balmer lines data reveal minima at the center of the plasma (maps not included here), for example, for a time delay of $1.5 \mu \mathrm{s}$ - illustrated in a recent publication (Parigger, 2019). These minima are representative of plasma expansion phenomena following initiation of optical breakdown. One can identify peak separation of the $\mathrm{H} \delta$ and $\mathrm{H} \beta$ lines, respectively. The $\mathrm{H} \delta$ map indicates that the background is shaded towards the UV, and there appear to be contributions from the $\mathrm{H} \epsilon$ line (at the low wavelenght side in the maps) just like in the recorded maps displayed in Fig. 1 . The $\mathrm{H} \delta$ line shows a shallower dip for higher electron density in the range of 0.1 to $1 \times 10^{17} \mathrm{~cm}^{-3}$. However, the $\mathrm{H} \beta$ peak separation indicates an electron density of $1.5 \times 10^{17} \mathrm{~cm}^{-3}$.

The Abel-inverted, $3.25 \mu$ s time-delay data display reasonably smooth line shapes for all four Balmer series lines. The $\mathrm{H} \delta$ peak separation can be easily demarcated, but the $\mathrm{H} \beta$ peak separation is difficult to extract. In other words, The $\mathrm{H} \delta$ line provides both PS and FWHM diagnostics as the electron density 

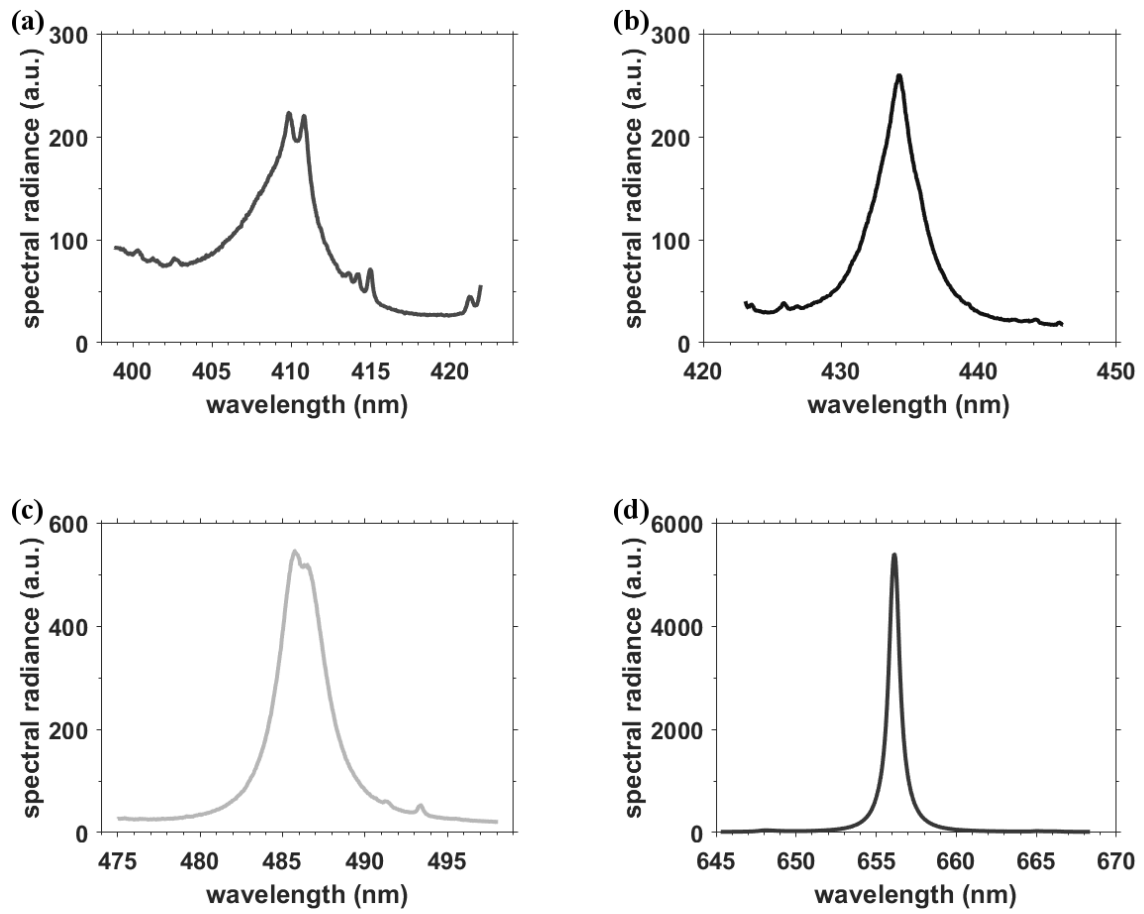

Figure 2. Average Balmer series hydrogen lines versus wavelength at $3-\mu$ s time delay, $0.025-\mu$ s gate. (a) $\mathrm{H} \delta$, (b) $\mathrm{H} \gamma$, (c) $\mathrm{H} \beta$, and (d) $\mathrm{H} \alpha$. The extra atomic lines, with magnitudes slightly larger than the background contributions, are due to nitrogen in the 1:1 hydrogen nitrogen mixture. In addition, the background contribution due to Bremsstrahlung radiation is larger for $\mathrm{H} \delta$ then for $\mathrm{H} \gamma$ and $\mathrm{H} \beta$, with minute contributions to $\mathrm{H} \alpha$.

decreases in the range of 0.1 to $1 \times 10^{17} \mathrm{~cm}^{-3}$. A 1 -nm peak separation at a time delay of $3.25 \mu$ s of the $\mathrm{H} \delta$ line implies $\mathrm{N}_{\mathrm{e}}=0.33 \times 10^{17} \mathrm{~cm}^{-3}$.

Abel-inverted maps usually indicate undulations of the signal along the radial direction. Such undulations can be expected as the laser-plasma expands - in related experiments of optical breakdown in air, multiple reflections can be seen in shadowgraphs that are captured following optical breakdown in air. Similar phenomena as depicted in the air shadowgraphs are expected to occur for the hydrogen:nitrogen mixture. Fig. 3 displays recorded air-breakdown shadowgraphs to further elucidate multiple reflections that are likely causing undulations in the plasma core. The shadowgraph experiments recorded 125 images for each time delay, the selected images portray typical images. 
(a)

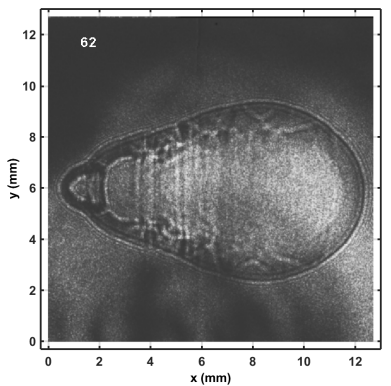

(b)

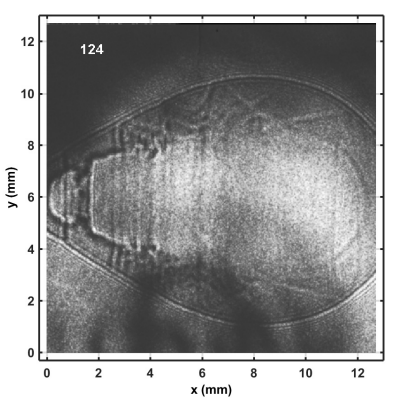

Figure 3. Shadowgraph of optical breakdown in laboratory air. The laser beam propagates from the right, images are captured using a second, imaging laser. The "bubble" on the laser side indicates absorption of laser radiation. Time delay: (a) $1.5 \mu \mathrm{s}$, image 62. (b) $3 \mu \mathrm{s}$, image 124 .

\subsection{Electron temperature and density}

Analysis of the recorded spectra along the slit height aims to evaluate electron temperature, $\mathrm{T}_{\mathrm{e}}$, from Boltzmann plots. Moreover, electron density, $\mathrm{N}_{\mathrm{e}}$, determination relies on formulae and tables that describe Stark broadening in laser-plasma. Fig. 4 portrays a typical Boltzmann-plot from line-of-sight measurements at $3-\mu$ s time delay. The Boltzmann plot method utilizes Boltzmann distributions and determination of the slope to extract the temperature from the negative of $1 /$ slope. The intercept of the straight line is not used for evaluation of the temperature. For data points from the four Balmer series members close to the fitted line, one can infer local thermodynamic equilibrium. As data points deviate from the fitted line, deviations from equilibrium may be concluded - self-absorbed lines would also cause deviations of data points from the straight line, and equally, ambiguities in determination of the baseline. However, expansion dynamics may lead to variations in the integrated line intensities as well. Fig. 4 is obtained from the areas of the four Balmer series lines with area ratio area- $\mathrm{H} \alpha: \mathrm{H} \beta: \mathrm{H} \gamma: \mathrm{H} \delta$ equals 1.5:0.42:0.19:0.13. Fig. 4 also shows error bars due to baseline uncertainties. The width ratio $\mathrm{H} \alpha: \mathrm{H} \beta: \mathrm{H} \gamma: \mathrm{H} \delta$ amounts to $0.84: 2.6: 2.7: 5.2$, and the $\mathrm{H} \beta$ and $\mathrm{H} \delta$ peak separations are 0.74 and $1.1 \mathrm{~nm}$, respectively. The electron densities inferred from the peak separations amount to $0.38 \times 10^{17} \mathrm{~cm}^{-3}$ and are consistent within error margins with electron densities obtained from the widths. Noteworthy, the uncertainties in the area and width determinations lead to estimated uncertainties of the electron density that are of the order of 10 to 20 per cent, equally for the electron density values computed from peak-separations. Automated analysis of the line-of-sight recorded data is further elaborated in Section 3.5, see Fig. 6. 


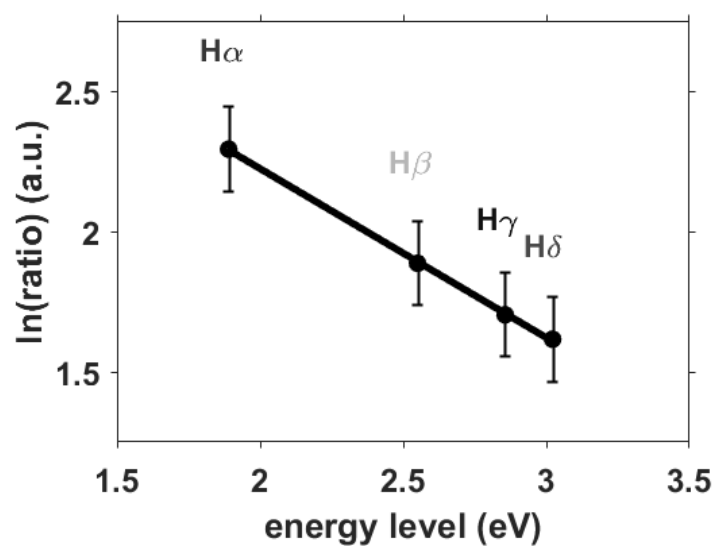

Figure 4. Electron temperature of $19.2 \mathrm{kK}$ inferred from line-of-sight data at $4.8 \mathrm{~mm}$ slit height, $3-\mu$ s time delay, $0.025-\mu$ s gate.

The electron density is related to the hydrogen Balmer series FWHM to the power of the order of 1.5, however, detailed theory predictions and experimental results indicate slight deviations. Empirical equations are obtained from log$\log$ fitting of FWHM and electron density. For red-shift of the $\mathrm{H} \alpha$ line, the dependency on electron density is almost linear. As mentioned earlier, Stark broadening widths increase from $\mathrm{H} \alpha$ to $\mathrm{H} \gamma$. The use of $\mathrm{H} \beta$ peak-separation for $\mathrm{N}_{\mathrm{e}}$ determination in laser-plasma adds a new diagnostic tool. Investigations of the $\mathrm{H} \delta$ peak separation are communicated in this work for $\mathrm{N}_{\mathrm{e}}$ in the range of 0.1 to $1 \times 10^{17} \mathrm{~cm}^{-3}$ and applied for the hydrogen-nitrogen laser plasma.

For the 3.25- $\mu$ s time delay, electron densities largely agree when inferred from the widths of the four Balmer series lines and from the peak-separations of $\mathrm{H} \beta$ and $\mathrm{H} \delta$. However, there are subtle differences of $\mathrm{N}_{\mathrm{e}}$ determined at $1.5 \mu$ s time delay. One may conclude from the appearance of the measured emission spectra and the computed Abel-inverted spectra that super- to hyper- sonic laser-plasma expansion speeds affect the establishment of local thermodynamic equilibrium. Details are investigated in the next section. Tables 1 and 2 summarize a snapshot of the data analysis.

Table 1 displays measured FWHM and peak-separations. The values are determined from the spectra at a slit height of $4.8 \mathrm{~mm}$ by using Matlab ${ }^{\circledR}$ software "peakfit.m" (O'Haver, 2018). Table 2 reports the electron densities.

\subsection{Plasma expansion dynamics}

Laser-plasma generation with laser pulses of the order of $10 \mathrm{~ns}$ and pulse energies of 80 to $800 \mathrm{~mJ}$ in ambient laboratory conditions causes a shock wave that 
Table 1. First four Balmer series FWHM and $\mathrm{H} \delta$ and $\mathrm{H} \beta$ peak-separations from line-of-sight data at $4.8-\mathrm{mm}$ slit height.

\begin{tabular}{ccccccc}
\hline \hline$\tau[\mu \mathrm{s}]$ & $\Delta \lambda_{\delta}[\mathrm{nm}]$ & $\Delta \lambda_{\delta-\mathrm{ps}}[\mathrm{nm}]$ & $\Delta \lambda_{\gamma}[\mathrm{nm}]$ & $\Delta \lambda_{\beta}[\mathrm{nm}]$ & $\Delta \lambda_{\beta-\mathrm{ps}}[\mathrm{nm}]$ & $\Delta \lambda_{\alpha}[\mathrm{nm}]$ \\
\hline 1.50 & $7.5 \pm 1.5$ & $1.4 \pm 0.8$ & $5.0 \pm 0.2$ & $4.2 \pm 0.25$ & $1.2 \pm 0.2$ & $1.17 \pm 0.1$ \\
3.25 & $5.8 \pm 0.3$ & $1.1 \pm 0.2$ & $3.2 \pm 0.2$ & $2.6 \pm 0.25$ & $0.8 \pm 0.15$ & $0.78 \pm 0.1$ \\
\hline \hline
\end{tabular}

Table 2. First four Balmer series electron densities, $\mathrm{N}_{\mathrm{e}}\left[10^{17} \mathrm{~cm}^{-3}\right]$, from line-of-sight data at $4.8-\mathrm{mm}$ slit height.

\begin{tabular}{cccccccc}
\hline \hline$\tau[\mu \mathrm{s}]$ & $\mathrm{N}_{\mathrm{e}}(\delta)$ & $\mathrm{N}_{\mathrm{e}}(\delta-\mathrm{ps})$ & $\mathrm{N}_{\mathrm{e}}(\gamma)$ & $\mathrm{N}_{\mathrm{e}}(\beta)$ & $\mathrm{N}_{\mathrm{e}}(\beta-\mathrm{ps})$ & $\mathrm{N}_{\mathrm{e}}(\alpha)$ & average $\mathrm{N}_{\mathrm{e}}$ \\
\hline 1.50 & 0.65 & 0.56 & 0.77 & 0.91 & 0.88 & 0.84 & 0.77 \\
3.25 & 0.44 & 0.38 & 0.41 & 0.46 & 0.46 & 0.45 & 0.43 \\
\hline \hline
\end{tabular}

expands at a rate of one to a few $\mathrm{mm} \mu \mathrm{s}^{-1}$, or a few $\mathrm{km} \mathrm{s}^{-1}$, give or take for time delays of the order of $1 \mu \mathrm{s}$. Early in the plasma decay, expansion speeds of up to $80 \mathrm{~km} \mathrm{~s}^{-1}$ are usually encountered that may be described in engineering terms as well above re-entry speeds or high hypersonic speeds. Several dozens of microseconds after plasma initiation, the shock wave reduces to the speed of sound.

In view of the "bubble" expanding in air and the associated isentropic expansion, one can determine electron density or temperature to elucidate the phenomena. Captured shadowgraphs can guide time-resolved spectroscopy, however, there should be an indication of the shockwave in spatially- and temporallyresolved measured spectra. One approach may utilize Radon- and Abel- inversion techniques (Pretzler et al., 1992) that are generally known as computed tomography methods. Alternatively, one may closely investigate the captured spectra along the slit height. Fig. 5 compares electron density results for a time delay of $3.0 \mu \mathrm{s}$. Increases in electron density at the plasma edges are indications of the expanding shock wave.

Systematic determination of the Stark widths from line-of-sight data is expected to reveal higher electron density near the shock wave than in the plasma core. Alternatively, determination of the area of the atomic Balmer series lines should indicate higher temperature near the shock wave than in the plasma center. Moreover, for time delays of several $\mu \mathrm{s}$ - as the shockwave expanded beyond the interrogated volume - the plasma is expected to be homogeneous and indicate local thermodynamic equilibrium for electron densities in excess of $10^{16} \mathrm{~cm}^{-3}$, as indicated by the necessary McWhirter criterion (McWhirter, 1965), and is expected to show higher temperature in the core.

The shock wave radius as function of time delay, $\tau$, is evaluated with the Taylor-Sedov formula for spherical expansion (Campanella et al., 2019),

$$
R(\tau)=\left(E_{p} / \rho \tau^{2}\right)^{1 / 5}
$$



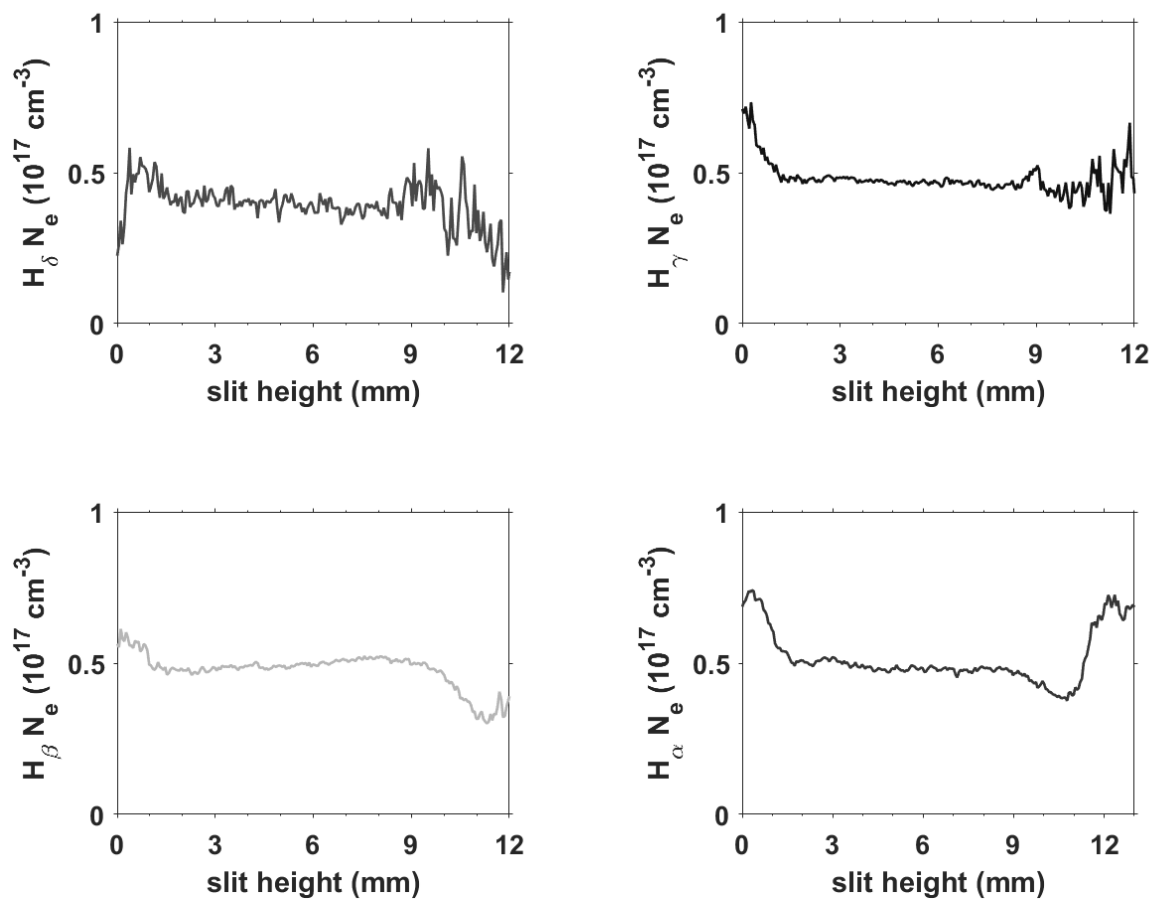

Figure 5. Electron density from line-of-sight data displayed in Fig. 1 at $3-\mu$ s time delay, 0.025- $\mu$ s gate. (a) $\mathrm{H} \delta$, (b) $\mathrm{H} \gamma$, (c) $\mathrm{H} \beta$, and (d) $\mathrm{H} \alpha$.

where the laser-pulse energy, $E_{p}$, for the experiments amounts to $0.15 \mathrm{~J}$, and the density of the 1:1 nitrogen hydrogen mixture, $\rho$, equals $0.37 \mathrm{~kg} \mathrm{~m}^{-3}$. For $\tau=3 \mu \mathrm{s}$, one finds for the radius $R=5.2 \mathrm{~mm}$. Fig. 6 illustrates the spectroscopic snapshot of the determined temperature profile for $\tau=3 \mu \mathrm{s}$.

Higher temperature is measured at slit-heights near $1.1 \mathrm{~mm}$ and $11.5 \mathrm{~mm}$ than in the central region, indicating the expanding shock wave. The predicted diameter of $10.4 \mathrm{~mm}$ for spherical expansion is identical with the diameter extracted from the spectra. Recording of shadowgraphs, see Fig. 3, indicates development of a largely symmetric bubble as time delay progresses beyond $1 \mu \mathrm{s}$. While the displayed shadowgraphs are captured in ambient laboratory air, shock waves propagate about a factor of four faster in hydrogen than in air, thereby evolving in hydrogen into spherical appearance faster than in air. Furthermore, the reductions in temperature near $1.5 \mathrm{~mm}$ and $10 \mathrm{~mm}$ are also expected for expanding shockwaves that propagate at speeds well above the speed of sound. The absolute error margins of the temperature are estimated to be of the order of $25 \%$ due to ambiguities in determining the background contributions to the 


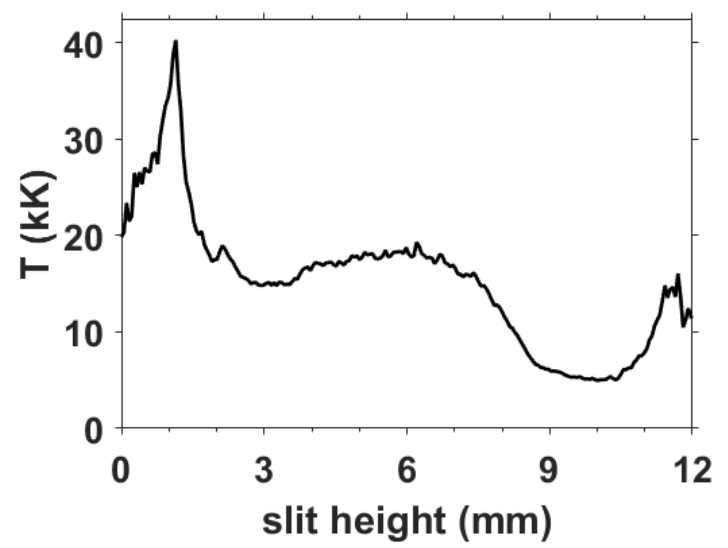

Figure 6. Electron temperature from line-of-sight data, $3-\mu$ s time delay, $0.025-\mu$ s gate. Average temperature: $15.4 \mathrm{kK}$.

line areas of the four Balmer series lines, yet the relative trends are consistent with expansion dynamics. The average temperature of the order of $15 \mathrm{kK}$ is also consistent with previous measurements of average temperatures. However, as the shock wave expands, both density and temperature variations occur near the shock front.

\section{Stellar astrophysical spectra}

Well-known white dwarfs near the earth are Sirius B and Procyon B. Sirius B shows a temperature of $26 \mathrm{kK}$, and absorption spectra in the visible part of the electromagnetic spectrum are composed of hydrogen Balmer series lines. In turn, Procyon B at a temperature of $7.9 \mathrm{kK}$ reveals molecular carbon Swan absorptions. Fig. 7 illustrates (Parigger et al., 2018) recorded spectra (Giammichele et al., 2012) that are accessible from the Montreal data-base (Dufour et al., 2019).

The spectral resolution, $\lambda / \Delta \lambda$, equals 555 for the displayed Sirius B and Procyon B absorption spectra. Clearly, the Sirius B absorption spectra displays larger absorption of $\mathrm{H} \gamma$ than of $\mathrm{H} \beta, \mathrm{H} \delta$ than $\mathrm{H} \gamma$, etc. In turn, laboratory emission spectra display the usual Boltzmann factor dependency, i.e., the integrated emission of $\mathrm{H} \beta$ is larger than that $\mathrm{H} \gamma, \mathrm{H} \gamma$ is larger than $\mathrm{H} \delta$, etc. The key considerations for reconciling the apparent ambiguity are evaluation of white-dwarf opacities. The bound-bound opacity corresponds to the emission profile, but the wavelength-dependent bound-free opacity increases towards higher members of the Balmer series. In essence, the bound-free opacity needs to be evaluated prior to comparison of recorded astrophysical data with laboratory results (Tremblay 
(a)

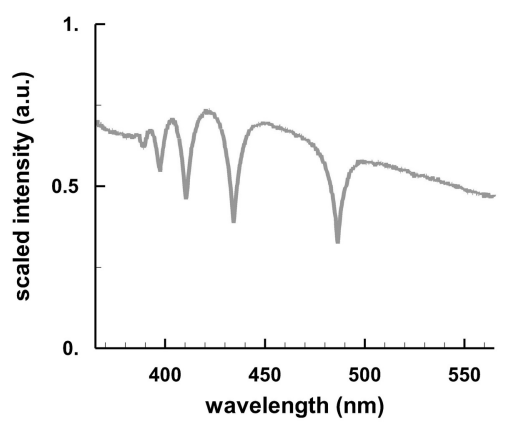

(b)

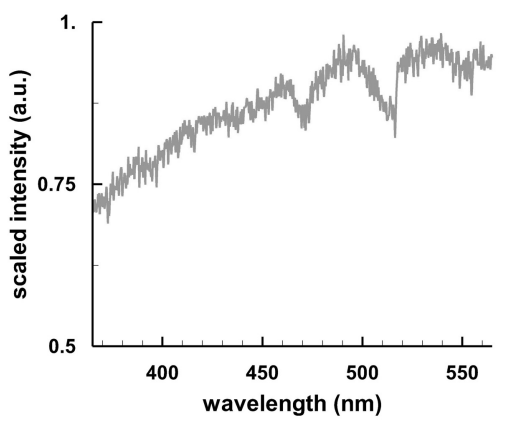

Figure 7. Sirius B and Procyon white dwarf spectra (Dufour et al., 2019): (a) Sirius $\mathrm{B}$ at $26 \mathrm{kK}$; and (b) Procyon $\mathrm{B}$ at $8 \mathrm{kK}$.

\& Bergeron, 2009). Conversely, as white dwarfs cool to the level of temperature seen for Procyon B, molecular absorption spectra can be demarcated. Carbon $\mathrm{C}_{2}$ Swan spectra may be developed more convincingly, such as for the white dwarf GJ 841 B (Dufour et al., 2019) that can be analyzed by fitting computed molecular spectra (Parigger et al., 2019b).

Collection of spectra from white dwarf stars preferably occurs with a resolving power sufficient for determination of the gravitational shift. Recent data for the white dwarf star GD 394 B, recorded with an echelle spectrometer on November 15, 2015, with KOA-ID: HI.20151115.19364 (Keck Observatory Archive, 2015), shows a resolving power of $\mathrm{R}=38000$, or a resolution of $\Delta \lambda=0.013 \mathrm{~nm}$.

Fig. 8(a) illustrates five overlapped regions of the recorded echelle spectra together with broad and narrow Lorentzian fits. Fig. 8(b) shows the expanded region of the central absorption. Both broad and narrow features of a WD astrophysical plasma are usually understood as absorptions from an outer region of the WD photosphere.

Analysis of the GD $394 \mathrm{~B}, \mathrm{H} \beta$ spectra (Parigger et al., 2019a) shows narrow and broad photosphere absorption-widths of $0.39 \mathrm{~nm}$ and $7.3 \mathrm{~nm}$ that would indicate electron densities of 0.032 and $2.0 \times 10^{17} \mathrm{~cm}^{-3}$, respectively. The electron density is determined from formulae that are valid for local thermodynamic equilibrium and that fulfil the necessary McWhirter criterion. Strictly speaking, then, the 0.39 -nm width implies an electron density indicative of non-equilibrium conditions. In view of the astrophysical WD plasma, one may conclude that there is a rather broad, likely in local thermodynamic equilibrium WD photosphere yielding a broad line profile, and another region that is significantly less dense. 
(a)

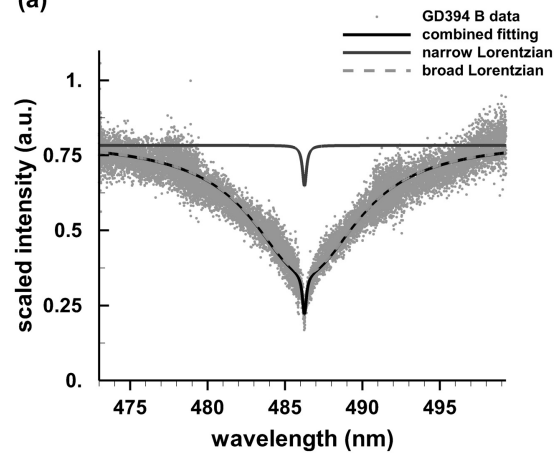

(b)

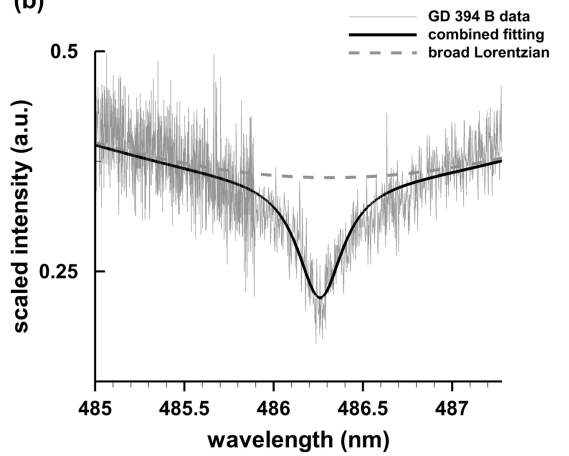

Figure 8. White dwarf GD $394 \mathrm{~B}, \mathrm{H} \beta$ combined absorption profiles, recorded with the HIRES echelle spectrometer, KOA-ID: HI.20151115.19364 (Keck Observatory Archive, 2015). (a) Fitting with two Lorentzians and (b) Expanded region (Parigger et al., 2019a).

Analysis of the white dwarf HG 7-85 from the Hyades cluster (Zuckerman et al., 2013) yields consistent results (Parigger et al., 2018) for broad Lorentzian center wavelength and gravitational shifts of $0.072 \mathrm{~nm}$ and $0.08 \mathrm{~nm}$, respectively.

\section{Discussion}

Laboratory experiments are essential in establishing detailed appearances of Balmer series line shapes for analysis of observed white-dwarf spectra. Modeling of astrophysical spectra however involves fairly detailed computations of the radiative transfer equation that is also known as the Boltzmann transport equation. The emission spectra of specifically the $\mathrm{H} \beta$ and $\mathrm{H} \gamma$ lines reveal two peaks at line center as expected from treatments of the Stark effect in hydrogen. A spectral resolutions of the order of 5,000 is sufficient for capturing Balmer series members with time-resolved spectroscopy when using a crossed Czerny-Turner spectrometer and an intensified charge-coupled device. Typical spectral resolutions of the order of 50,000 are available with echelle spectrometers that are in use at observatories for continuous recording of astrophysical plasma from white dwarfs. Comparisons of laboratory spectra then requires adding individually recorded time-resolved spectra to obtain an average for comparison with white dwarf spectra. In other words, averaging of time-resolved spectra corresponds to averaging data for different electron densities. Consequently, details seen in the laboratory are absent in observed white dwarf spectra. Analysis of the white-dwarf spectra with at least two Lorentzians would indicate that at 
least two regions indeed occur with different electron densities. The explored $\mathrm{H} \gamma$ and $\mathrm{H} \delta$ lines can provide additional diagnostics for white dwarfs, especially in the range of 0.1 to $1 \times 10^{17} \mathrm{~cm}^{-3}$. However, bound-free opacity effects would need to be included when using, say, the first four lines of the Balmer series. Analysis of white-dwarfs and other astrophysical objects such as active galactic nuclei from only one line, i.e., $\mathrm{H} \beta$ may be preferred as bound-free effects may not be significant across one line and for purposes of determining widths and asymmetries of stellar $\mathrm{H} \beta$ lines. Future work however should include new results from laboratory measurements that address specifically the shapes of lines used for diagnosis of astrophysical spectra.

Acknowledgements. The author appreciates the support in part by the Center for Laser Application, a State of Tennessee funded Accomplished Center of Excellence at the University of Tennessee Space Institute.

\section{References}

Campanella, B., Legnaioli, S., Pagnotta, S., Poggialini, F., Palleschi, V. Shock Waves in Laser-Induced Plasmas. 2019, Atoms, 7, 57

Dufour, P., Blouin, S., Coutu, S., Fortin-Archambault, M., Thibeault, C., Bergeron, P., Fontaine, G. The Montreal White Dwarf Database: A Tool for the Community. Available online: http://dev.montrealwhitedwarfdatabase.org (accessed on 3 June 2019)

Giammichele, N., Bergeron P., Dufour, P. Know your neighborhood: A detailed model atmosphere analysis of nearby white dwarfs. 2012, Astrophys. J. Supp. Ser., 199, $29-63$

Gigosos, M. A., González, M. Á., Cardeñoso, V. Computer simulated Balmer-alpha, -beta and -gamma Stark line profiles for non-equilibrium plasmas diagnostics. 2003, Spectrochim. Acta B At. Spectrosc., 58, 1489-1504

Greene, Jr., R. E. 1961, The Shift and Shape of Spectral Lines, Pergamon Press: Oxford, UK, 1961, Chapter 4, pp. 118-193

Griem, H. R. 1974, Spectral Line Boradening by Plasmas, Academic Press: Cambridge, $M A, U S A, 1974$

Halenka, J., Vujićić, B., Djurović, S. Shift of the peaks of the $\mathrm{H} \beta$ spectral line. 1989, J. Quant. Spectrosc. Radiat. Trans., 42, 571-573

Halenka, J., Olchawa, W., Madej, J., Grabowski, B. Pressure shift and gravitational redshift of Balmer lines in white dwarfs: Rediscussion. 2015, Astrophys. J., 808, $131-140$

Holtsmark, J. Über die Verbreiterung von Spectrallinien. 1919, Ann. Phys., 58, 577630

Inglis, D. R., Teller, E. Ionic depression of series limits in one-electron spectra. 1939, Astrophys. J. 90, 439-448 
Ivković, M., Konjević, N., Pavlović, Z. Hydrogen Balmer beta: The separation between line peaks for plasma electron density diagnostics and self-absorption test. 2015, J. Quant. Spectrosc. Radiat. Trans., 154, 1-8

W. M. Keck Observatory Archive (KOA). Available online: https://koa.ipac.caltech.edu (accessed on 5 May 2018)

Kunze, H.-J. 2009, Introduction to Plasma Spectroscopy, Springer: New York, NY, USA, 2009

McWhirter, R. W. P. Spectral Intensities. 1965, Plasma Diagnostic Techniques; Huddlestone, R.H., Leonard, S.L., Eds.; Academic Press: New York, NY, USA, 1965; pp. 201-264

Mijatović, Z., Pavlov, M., Djurović, S. Shift of the $\mathrm{H} \beta$ line in dense hydrogen plasmas. 1991, Phys. Rev. A, 43, 6095-6097

Miziolek A. W., Palleschi V., Schechter, I., Eds. 2006, Laser Induced Breakdown Spectroscopy, Cambridge University Press: New York, NY, USA, 2006

O'Haver T. C. Command-line peak fitter for time-series signals. Version 9.0, January 2018, Mathworks File Exchange. Available online: https://www.mathworks.com/matlabcentral/fileexchange/23611-peakfit-m (accessed June 4 2019)

Oks, E. 2017, Diagnostics of Laboratory and Astrophysical Plasmas Using Spectral Lineshapes of One-, Two-, and Three-Electron Systems, World Scientific: Singapore, 2017; pp. 147

Parigger, C. G., Woods, A. C., Witte, M. J., Swafford, L. D., Surmick, D. M. Measurement and Analysis of Atomic Hydrogen and Diatomic Molecular AlO, $\mathrm{C}_{2}, \mathrm{CN}$, and TiO Spectra Following Laser-induced Optical Breakdown. 2014, J. Vis. Exp., 84, e51250

Parigger, C. G., Drake, K. A., Helstern, C. M., Gautam, G. Laboratory HydrogenBeta Emission Spectroscopy for Analysis of Astrophysical White Dwarf Spectra. 2018, Atoms, 6, 36

Parigger, C. G. Measurements of Gaseous Hydrogen-Nitrogen Laser-Plasma. 2019, Atoms, 7, 61

Parigger, C. G., Helstern, C. M., Gautam, G., Drake, K. A. Atomic and molecular line shapes in laboratory and selected astrophysical plasma. 2019, J. Phys.: Conf. Ser., in press

Parigger, C. G., Helstern, C. M., Gautam, G. Laser-Plasma and Self-Absorption Measurements with Applications to Analysis of Atomic and Molecular Stellar Astrophysics Spectra. 2019, Atoms, 7, 63

Pretzler, G., Jäger, H., Neger, T., Philipp, H., Woisetschläger, J. Comparison of Different Methods of Abel Inversion Using Computer Simulated and Experimental Side-On Data. 1992, Z. Naturforsch., 47a, 955-970

Schrödinger, E. Quantisierung als Eigenwertproblem. 1926, Ann. Phys. 385, 437-490

Singh, J. P., Thakur S. N., Eds., 2019, Laser Induced Breakdown Spectroscopy, Elsevier Science: New York, NY, USA, 2019 
Tennyson, J., Yurchenko, S. N. ExoMol: Molecular line lists for exoplanet and other atmospheres. 2012, Mon. Not. R. Astron. Soc., 425, 21-33

The Nobel Prize in Physics 1933. Available online: https://www.nobelprize.org/prizes/physics/1933/summary/ (accessed on May $242019)$

Tremblay, P.-E., Bergeron, P. Spectroscopic Analysis of DA White dwarfs: Stark broadening of hydrogen lines including nonideal effects. 2009, Astrophys. J., 696, 17551770

Wiese, W. L. Line Broadening. 1965, Plasma Diagnostic Techniques; Huddlestone, R.H., Leonard, S.L., Eds.; Academic Press, New York, NY, USA, 1965; pp. 265317

Zuckerman, B., Xu, S., Klein, B., Jura, A. Thy Hyades cluster: Identification of a planetary system and ascaping white dwarfs. 2013, Astrophys. J., 770, 140-141 